\title{
产 \\ Derecho e indicadores una perspectiva
}

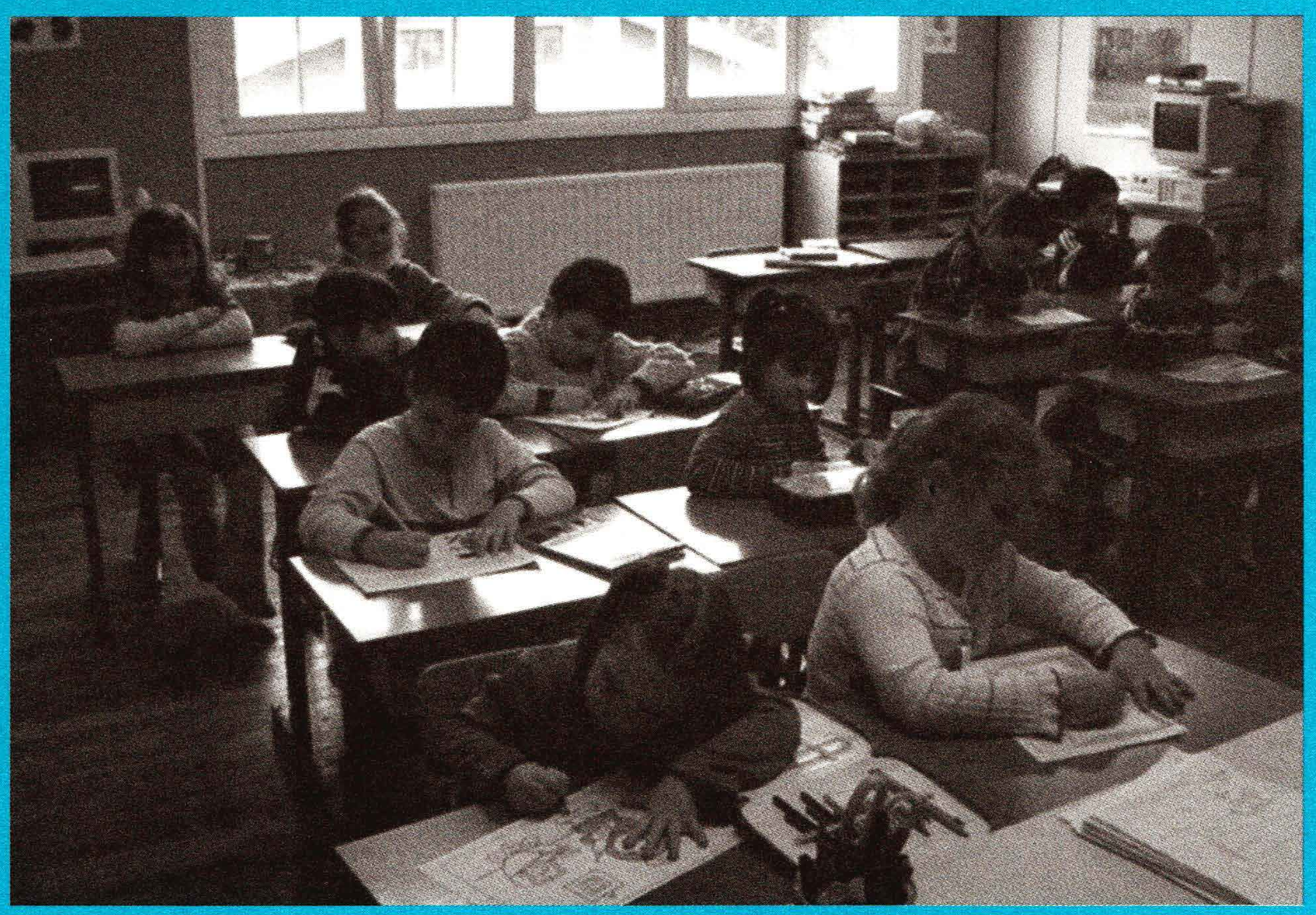




\section{Luis Eduardo Pérez Murcia}

El autor es economista de la Universidad Nacional de Colombia y magíster en Economía y especialista en Estadistica de la misma Universidad. Parte de su experiencia profesional la ha dedicado al estudio de las políticas educativas y al diseño de indicadores para monitorear el nivel de disfrute del derecho a la educación. Se desempeña actualmente como investigador del Centro de Estudios Derecho, Justicia y Sociedad.

\section{Resumen}

El artículo expone la necesidad de fortalecer los vínculos entre el diseño de indicadores educativos y el contenido del derecho a la educación, al tiempo que presenta un modelo de indicadores para monitorear y evaluar el nivel de disfrute de este derecho, Tomando como base el sistema de la $4 \mathrm{~A}$ propuesto por $\mathrm{K}$. Tomasevski, se propone un sistema de indicadores que permita dar cuenta del nivel de cumplimiento del Estado frente a las principales obligaciones en materia de asequibilidad, accesibilidad, aceptabilidad y adaptabilidad de la educación.

\section{Palabras clave} tabilidad.

Indicadores, derecho a la educación, monitoreo, disfrute, asequibilidad, accesibilidad, aceptabilidad, adap-

\section{Abstract}

The article exposes the need to enhance the relationship between the construction of educational indicators and the right to education. At the same time, it presents a new model of policy indicators that act as safeguards to monitor and asses the fulfillment of the right to education. Based on the $4 \mathrm{~A}$ scheme proposed by Katarina Tomasevski, the set of indicators proposed pretends to evaluate the level of commitment of the Colombian State to the fulfillment of this right in terms of availability, accessibility, acceptability and adaptability.

\section{Key words}

Indicators, right to education, monitoring, enjoyment, availability, accessibility, acceptability and adaptability. 
$\mathbb{P}$ ese a que existe un importante volumen de estadísticas educativas en Colombia ${ }^{1}$, y a que periódicamente se presentan análisis que dan cuenta de algunos aspectos de las problemáticas del sector, el país no dispone de informes sobre el nivel de realización del derecho a la educación Puntualmente, los estudios sobre educación tienden a concentrarse en aspectos como la cobertura, calidad y eficiencia y deserción del sistema educativo, sin que se tenga una mirada de conjunto que vincule las obligaciones del Estado frente al derecho a la educación y los logros alcanzados mediante la implementación de las políticas públicas educativas.

Por lo anterior, y considerando que con base en el Pacto Internacional de Derechos Económicos, Sociales y Culturales es deber del Estado informar sobre el nivel de avance en el disfrute del derecho a la educación, el presente artículo se ocupa de desarrollar una propuesta de indicadores que permita dar cuenta del nivel de disfrute de este derecho. El artículo está organizado en tres acápites. El primero

\footnotetext{
Este artículo sintetiza algunas de las reflexiones presentadas en el documento titulado "Modelo de indicadores para monitorear y evaluar las politicas educativas desde la perspectiva del derecho a la educación", elaborado por Luis Eduardo Pérez Murcia en el marco de la investigación Análisis comparado de las políticas educativas en Bogotá, dos ciudades colombianas y tres ciudades latinoamericanas: una mirada desde la perspectiva del derecho a la educación; contratada por el Instituto de Investigación Educativa y Desarrollo Pedagógico. El artículo recoge los aportes realizados por el equipo de investigación del Centro de Estudios Derecho, Justicia y Sociedad integrado por Rodrigo Uprimny, César Rodríguez, Mauricio Garcia, Camilo Castillo y Miquel Jordana, a quienes agradece el autor.
}

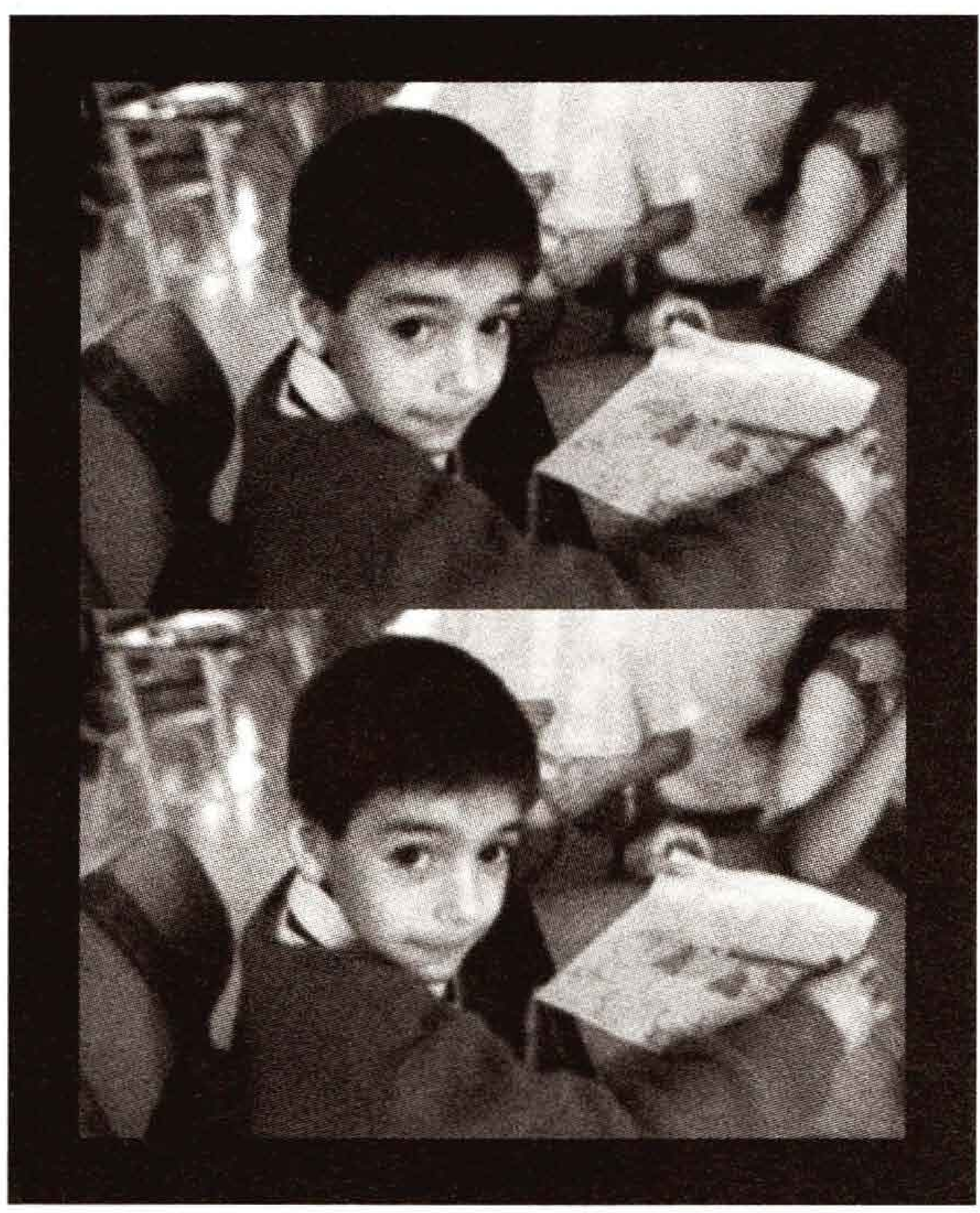

llama la atención sobre la importancia de fortalecer el vínculo entre las obligaciones del Estado en materia de derechos humanos y el diseño de indicadores. El segundo explica las características especiales de los indicadores elaborados desde la perspectiva de los derechos humanos. La última sección presenta un modelo de indicadores para monitorear y evaluar el nivel de disfrute del derecho a la educación.

\section{Derecho e indicadores: el ejercicio de fortalecer un vínculo natural}

Los sistemas de indicadores de educación existentes enfrentan limitaciones para establecer el nivel en que las políticas públicas educativas aportan a la realización del derecho a la educación de todos y todas, sin discriminación alguna; en especial, de los sujetos y grupos poblacionales tradicionalmente ex- 
cluidos y marginados de los procesos de desarrollo. Por lo mismo, y adoptando como punto de partida un enfoque de políticas públicas cuyo diseño, implementación y evaluación estén guiadas por el contenido de los derechos humanos y las obligaciones del Estado, es posible argumentar que, dado que el objetivo último de toda política pública educativa deberá ser la promoción del derecho a la educación, es indispensable reenfocar los indicadores educativos desde una perspectiva de derechos humanos. Expresado en otros términos, es necesario fortalecer el vínculo natural existente entre las obligaciones del Estado en materia

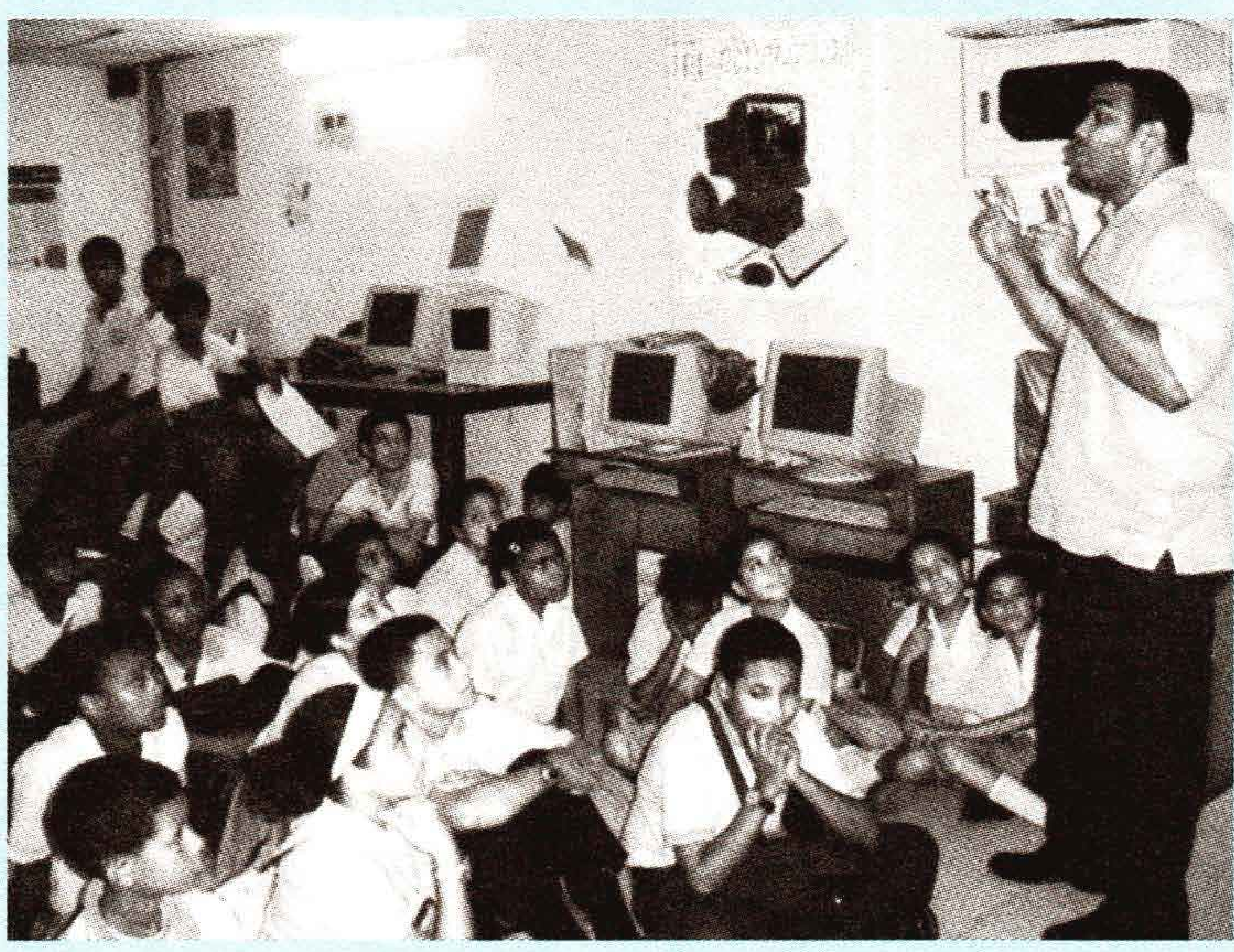
de derechos humanos y el diseño de indicadores, para dar cuenta de su cumplimiento ${ }^{2}$.

Puntualmente, es imperativo que los indicadores para el seguimiento y monitoreo de la política educativa permitan evidenciar en qué medida el Estado cumple con sus obligaciones de respeto, protección y promoción del derecho a la educación. En este sentido, y conforme lo dispone la Observación General No. 3 del Comité de Derechos Económi-

2 Esta postura es coherente con el enfoque de desarrollo humano, en el sentido de que, desde sus planteamientos generales, es posible deducir que el objetivo de toda política pública es contribuir al ejercicio de los derechos humanos de todos y todas, en especial de los sujetos y grupos más desprotegidos. Véase Informe sobre Desarrollo Humano 2005. La cooperación internacional ante una encrucijada: ayuda al desarrollo, comercio y seguridad en un mundo desigual. Programa de las Naciones Unidas para el Desarrollo. Ediciones Mundi-Prensa. 2005.

cos, Sociales y Culturales, relativa al carácter de las obligaciones de los Estados Parte en materia de derechos económicos, sociales y culturales, es menester que los indicadores permitan establecer de qué manera el Estado adopta todas las medidas posibles, hasta el máximo de los recursos disponibles, para hacer efectiva la realización del derecho a la educación de todos y todas.

A propósito, cabe recordar que con respecto al Pacto Internacional de Derechos Económicos, Sociales y Culturales, la Observación General No. 3 establece que los Estados deben disponer del máximo de los recursos necesarios para adoptar medidas legislativas, administrativas, judiciales, económicas, sociales y educacionales, para asegurar, de forma progresiva, y sin discriminación alguna, el pleno ejercicio del derecho a la educación. Tales providencias comprenden el 
respeto por el derecho a la educación de todos y todas; la adopción de medidas positivas para facilitar y proveer el ejercicio del derecho a la educación, y la garantía de mecanismos para proteger el ejercicio de este derecho, de manera que se evite que terceros puedan obstaculizarlo.

En armonía con las disposiciones prescritas en la Observación General citada, se requiere que los indicadores para el monitoreo de las políticas educativas permitan evidenciar los siguientes aspectos relacionados directamente con el derecho a la educación ${ }^{3}$ :

- Determinar si el Estado está cumpliendo, y el grado en que lo está haciendo, sus obligaciones de respeto, protección y promoción del derecho a la educación.

- Determinar si el diseño e implementación de las políticas públicas educativas es coherente con el sentido de las obligaciones del Estado: no discriminación, no "regresividad", y adopción de medidas concretas, con recursos suficientes para la realización del derecho a la educación ${ }^{4}$.

\footnotetext{
Defensoría del Pueblo. Sistema de seguimiento y evaluación de la politica educativa a la luz del derecho a la educación. Investigación realizada por Luis Eduardo Pérez Murcia. Bogotá, Colombia. 2004. Pág. 64.
}

\footnotetext{
4 Al respecto puede consultarse Comité de Derechos Económicos, Sociales y Culturales, Observación General No. 3, relativa a la indole de las obligaciones de los Estados Parte.
} 

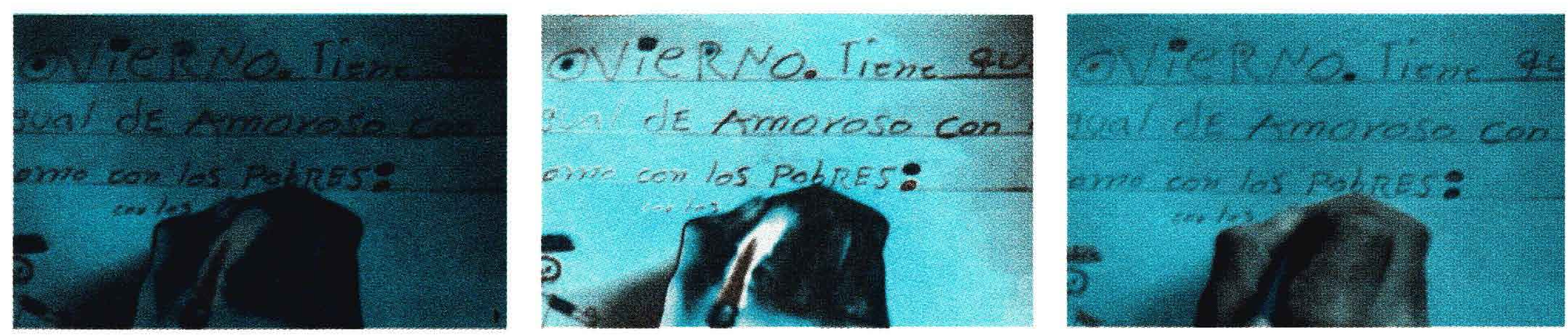

- Disponer de información que oriente el diseño y ejecución de políticas públicas educativas, fundamentadas en la realización del derecho a la educación.

\section{Especificidad de los indicadores con perspectiva de derechos humanos}

A tono con experiencias nacionales e internacionales de investigación en materia de indicadores educativos desde la perspectiva de los derechos humanos -en especial, el trabajo adelantado por la Comisión de Derechos Humanos de Sudáfrica, que, por mandato constitucional, diseñó en 1996 un sistema de indicadores para monitorear y evaluar la realización de los derechos sociales-, con el sistema de las 4-A, propuesto por la profesora Katarina Tomasevski, relatora especial de las Naciones Unidas para el Derecho a la Educación 1998-2004, y con el Sistema de Seguimiento y Evaluación de Políticas Públicas desarrollado por la Defensoría del Pueblo de Colombia, con el fin de determinar el nivel de realización de los derechos económicos, sociales y culturales en el país, es posible concluir que existen, por lo menos, tres criterios centrales de diferenciación entre los indicadores educativos tradicionales y los indicadores elaborados en perspectiva de derechos humanos. Estos son el fundamento de la medida, la no discriminación y la no "regresividad" 5 .

En cuanto al primer criterio de diferenciación, el fundamento de la medida, cabe resaltar que, en tanto los indicadores educativos convencionales tienen, generalmente, como referente fundamental o razón de medida una meta establecida en un plan de desarrollo, el control del gasto público o la evolución del sector, los indicadores en perspectiva de derechos humanos responden, de manera estricta, al contenido del

5 Sobre las experiencias de investigación citadas, puede consultarse: Comisión de Derechos Humanos de Sudáfrica. Recuperado el 23 de octubre de 2005, de http://www.sahrc.org.za/4th_esr_chap_7.pdf; Tomasevski Katarina. Human Rights Obligations: Making Education Available, Accessible, Acceptable and Adaptable. Education Primers No. 3., Novum Grafiska AB, Gothernburg, Suecia, 2001; y, Defensoría del Pueblo. Sistema de seguimiento y evaluación de la política educativa a la luz del derecho a la educación. Investigación realizada por Luis Eduardo Pérez Murcia. Bogotá, Colombia. 2004. 
derecho a la educación y a las correlativas obligaciones del Estado derivadas de la Constitución Política, a los instrumentos internacionales de derechos humanos, a la jurisprudencia y doctrina de los órganos internacionales de protección, a la jurisprudencia de la Corte Constitucional y a las normas locales.

Conforme a estas normas, y en especial al esquema de organización del derecho a la educación en un sistema de 4-A, propuesto por la profesora Tomasevski y adoptado por el Comité de Derechos Económicos, Sociales y Culturales en la Observación General No. 13, el derecho a la educación comprende tres dimensiones, cuatro componentes y cuatro conjuntos de obligaciones, de los cuales se espera que los indicadores den cuenta.

Las tres dimensiones son: dimensión del derecho a la educación, que obedece a la naturaleza y alcance del derecho derivado de las normas nacionales e internacionales de protección y que envuelve tanto al derecho a la disponibilidad de educación -obligación de asequibilidad - como al derecho de acceso a la educación -obligación de accesibilidad-. La dimensión de los derechos en la educación, que hace referencia a la promoción y garantía de todos los derechos humanos en el ámbito de la escuela y que contempla el derecho a una educación aceptable obligación de aceptabilidad-. Y, finalmente, la dimensión de los derechos por la educación, que se refiere al papel de la educación como multiplicadora de los demás derechos humanos y que incluye tanto el derecho a una educa-

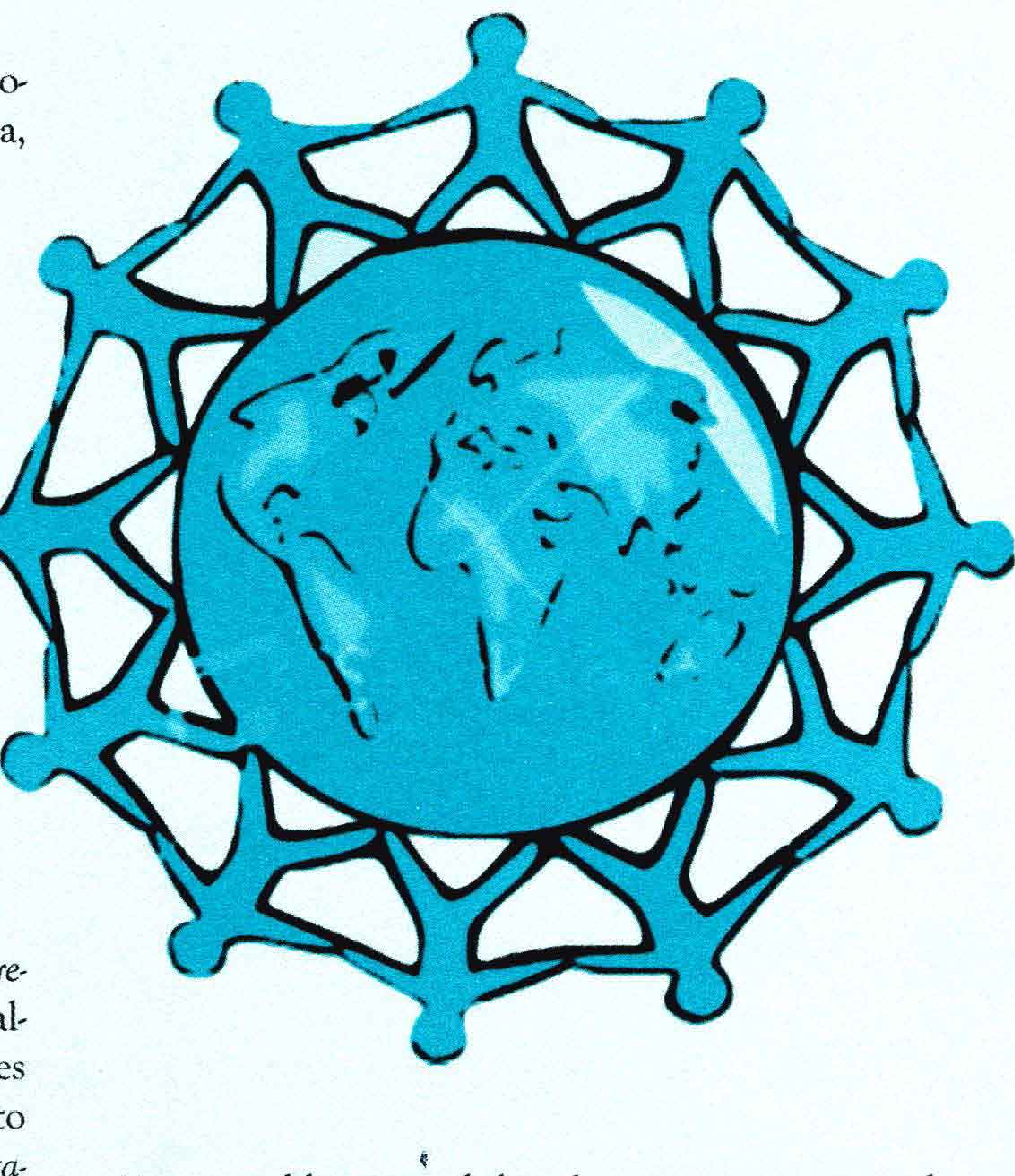

ción aceptable como el derecho a permanecer en el sistema educativo obligación de adaptabilidad ${ }^{6}$.

Finalmente, en torno a este primer criterio de diferenciación de los indicadores de derechos humanos, es oportuno mencionar que el hecho de que los indicadores elaborados desde la perspectiva de los derechos humanos contribuyan a monitorear el cumplimiento de obligaciones del Estado frente al derecho a

\footnotetext{
6 Una explicación detallada de los diferentes componentes del derecho, puede consultarse en Defensoria del Pueblo. Op. Cit., Págs. 34-38.
} 


\section{Asequibilidad}

\section{Accesibilidad}

Aceptabilidad

\section{Adaptabilidad}

Tabla 1: Sistema de las 4-A, propuesto por Katarina Tomasevski ${ }^{7}$

Escuelas

Profesores

Obligatoria

Post-obligatoria

Necesidades especiales

Niños y niñas por fuera

del sistema educativo
- Disponibilidad de escuelas.

- Libertad de fundar establecimientos educativos.

- $\quad$ Disponer de fondos para escuelas públicas.

- Disponer de fondos para escuelas privadas.

- Criterios de selección.

- $\quad$ Aptitudes para la enseñanza.

- Derechos laborales.

- Libertades sindicales.

- Responsabilidades profesionales.

- Libertades académicas.

- Acceso para todos y todas.

- Gratuidad.

- Asistencia escolar garantizada.

- Libertad de los padres y madres a elegir la educación de los niños y las niñas.

- Acceso sin discriminación.

- Acceso preferencial para los grupos vulnerables.

- Admisión basada en criterios académicos.

- Reconocimiento de diplomas extranjeros.

- Estándares mínimos de calidad.

- Respeto de la diversidad.

- Lenguaje de instrucción.

- Orientación y contenidos curriculares.

- Disciplina escolar.

- Derechos de los estudiantes.

- $\quad$ Niños y niñas con discapacidad.

- Niños y niñas trabajadores.

- Niños y niñas refugiados.

- Niños y niñas privados de la libertad.

7 Traducción libre del sistema presentado por Katarina Tomasevski . Op. Cit., P. 12. 
la educación, les permite gozar de cierto carácter de exigibilidad. Al respecto, el Informe sobre Desarrollo Humano 2000 destacó que los indicadores pensados desde los derechos humanos se convierten en instrumentos para alcanzar los siguientes objetivos ${ }^{8}$ :

- Formular mejores políticas y vigilar los progresos realizados.

- Determinar los efectos no deseados de leyes, politicas y prácticas.

- Determinar qué actores están influyendo en la realización de los derechos.

- Poner de relieve si esos actores están cumpliendo sus obligaciones.

- Advertir de antemano posibles violaciones y poder adoptar medidas preventivas.

- Fortalecer el consenso social respecto a decisiones difíciles que deban adoptarse frente a la limitación de recursos.

- Sacar a la luz cuestiones que han sido desatendidas o silenciadas.

8 Informe sobre Desarrollo Humano 2000. Derechos humanos y desarrollo humano, capítulo 5, Uso de indicadores para exigir responsabilidad en materia de derechos humanos, Nueva York, 2001.
En cuanto al segundo criterio de diferenciación de los indicadores convencionales y los indicadores elaborados en perspectiva de derechos, es oportuno señalar en primer lugar que la no discriminación es uno de los principios fundamentales de los derechos humanos. Según el párrafo $7^{\circ}$ de la Observación General No. 18 del Comité de Derechos Humanos, el término "discriminación" se debe entender como:

“... toda distinción, exclusión, restricción o preferencia que se basen en determinados motivos, tales como la raza, el color, el sexo, el idioma, la religión, la opinión política o de otra índole, el origen nacional o social, la posición económica, el nacimiento o cualquier otra condición social, y que tengan por objeto o por resultado anular o menoscabar el reconocimiento, goce o ejercicio, en condiciones de igualdad, de los derechos humanos y libertades fundamentales de todas las personas"

En segundo término, es preciso aclarar que, conforme al derecho internacional de los derechos 


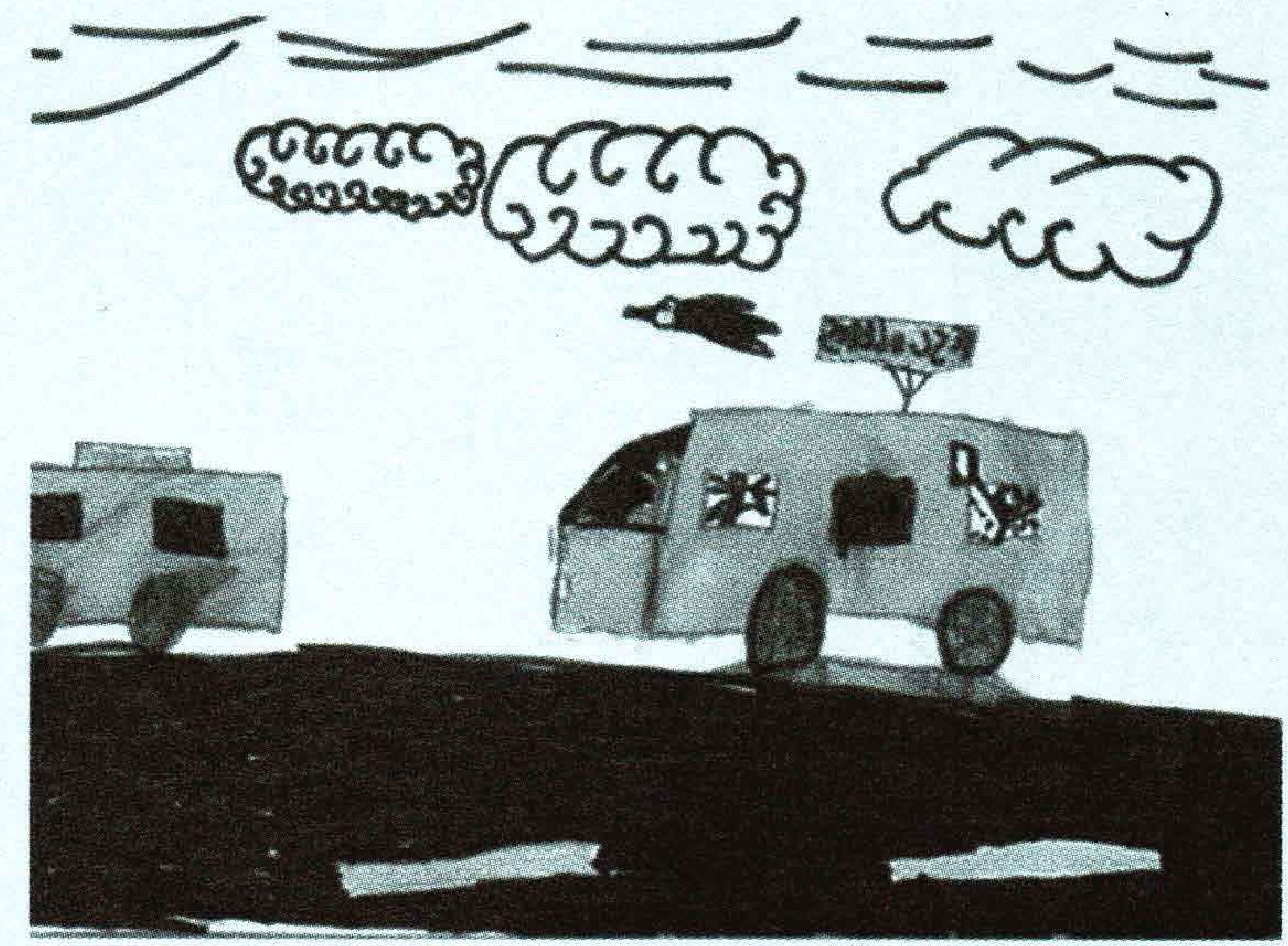

humanos, la no discriminación sólo se hace efectiva cuando se asegura el derecho al trato preferente a los sujetos y grupos poblacionales discriminados. En estricto sentido, negar el derecho al trato preferente -discriminación positiva - a un sujeto o grupo poblacional discriminado determina una conducta discriminatoria.

No obstante el carácter perentorio de la obligación a la no discriminación -lo cual exige valorar sus diferentes modalidades, impactos y efectos sobre la realización del derecho a la educación-, el diseño de metodologias para medir discriminación en los derechos sociales, en general, y en el derecho a la educación, en particular, tienen un desarrollo incipiente. Sólo es posible captar la discriminación, en cualquiera de sus formas $u$ expresiones, si se investigan sus causas más profundas y las formas en que se materializa. Por lo mismo, es necesario que los indicadores de derechos humanos incorporen, como una variable aproximada de la discriminación, un elemento de diferenciación, según grupos poblacionales. Esto permitirá realizar una primera aproximación al impacto diferencial de las políticas públicas educativas en los distintos grupos poblacionales e identificar cuáles de estos grupos se encuentran excluidos a causa, posiblemente, de politicas que, aunque no son abiertamente discriminatorias, producen impactos que pueden inducir a perpetuar las formas de discriminación existentes.

En este sentido, es importante que indicadores educativos como, por ejemplo, los indicadores de acceso, tipo, tasas brutas y netas de cobertura; los indicadores de calidad, como resultados de pruebas de saber e ICFES, y los indicadores de permanencia, como tasas de deserción y de retención, se desagreguen según los siguientes grupos poblacionales: población con discapacidad, población desplazada, población negra e indígena, minorías religiosas y personas con diferente orientación sexual. Además, al interior de estos grupos deben clasificarse los indicadores según género y grupos de edad.

A pesar de que este tipo de desagregaciones no permiten concluir, en rigor, si una política es discriminatoria o no, constituyen un primer paso para evidenciar fenómenos de discriminación. Así, por ejemplo, si se encontrara que la tasa de matrícula de un grupo poblacional es tres veces menor que la de otro, no se podría concluir que existe un fenómeno de discriminación en contra de este últi- 
mo. Sin embargo, se dispondría de información que permitiría aportar evidencias empíricas para una hipótesis de investigación en este sentido. De todas maneras, resulta claro que la validación rigurosa de la hipótesis requeriría elaborar, por lo menos, un estudio de caso en el que se indague hasta qué punto los diferenciales en la tasa de matrícula entre diferentes grupos poblacionales obedecen a un fenómeno discriminatorio.

Finalmente, en torno al tercer principio de diferenciación de los indicadores - la no "regresividad"-, el Comité de Derechos Económicos, Sociales y Culturales estableció que los Estados no pueden adoptar medidas que conduzcan a niveles de realización del derecho a la educación menores que los niveles alcanzados, independientemente de que los mismos estén por encima de los mínimos protegidos en las normas del Derecho Internacional de los Derechos Humanos. Para efectos de identificar el nivel de progreso y la no "regresividad", los indicadores educativos elaborados desde la perspectiva del derecho a la educación deben medir la evolución de las políticas, con especial atención en los progresos o retrocesos de los sujetos y grupos poblacionales tradicionalmente discriminados. Sobre el particular, cabe señalar que el nivel de progreso o "regresividad" sólo es factible de evaluar al captar los indicadores de manera periódica, mediante, por ejemplo, la construcción de series anuales con los indicadores

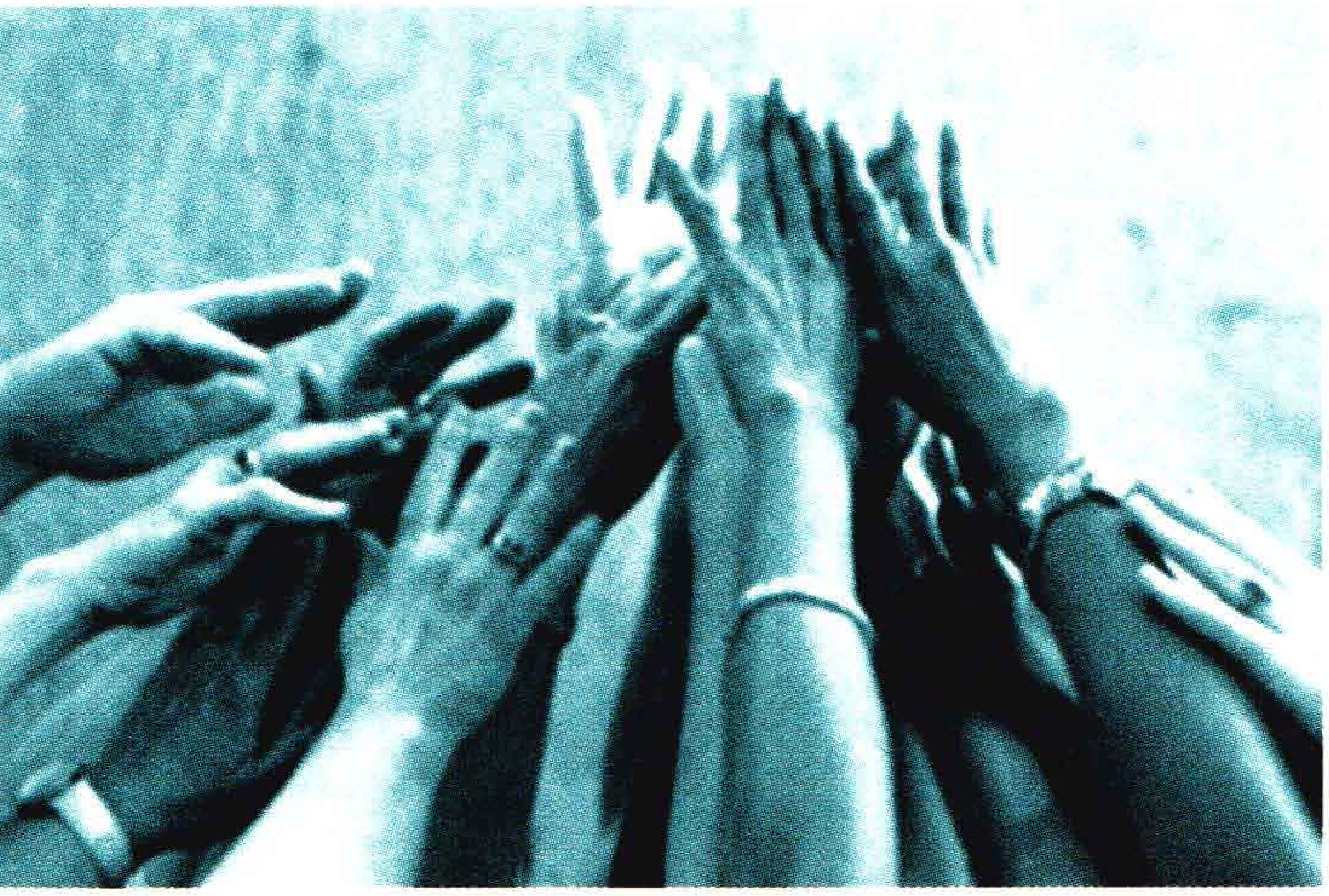

más representativos, para cada dimensión del derecho y las correlativas obligaciones del Estado.

\section{Modelo de indicadores educativos para monitorear y evaluar el nivel de realización del derecho a la educación}

Conforme a los principios que guían los indicadores de derechos humanos expresados en el acápite anterior, se propone a continuación un sistema de indicadores que permita dar cuenta del nivel de cumplimiento del Estado frente a las principales obligaciones en materia de asequibilidad, accesibilidad, aceptabilidad y adaptabilidad de la educación.

Para la selección de los indicadores convencionales del sector educativo a ser incorporados en el sistema, y el desarrollo de nuevos indicadores para las obligaciones que no tienen indicadores asocia- 


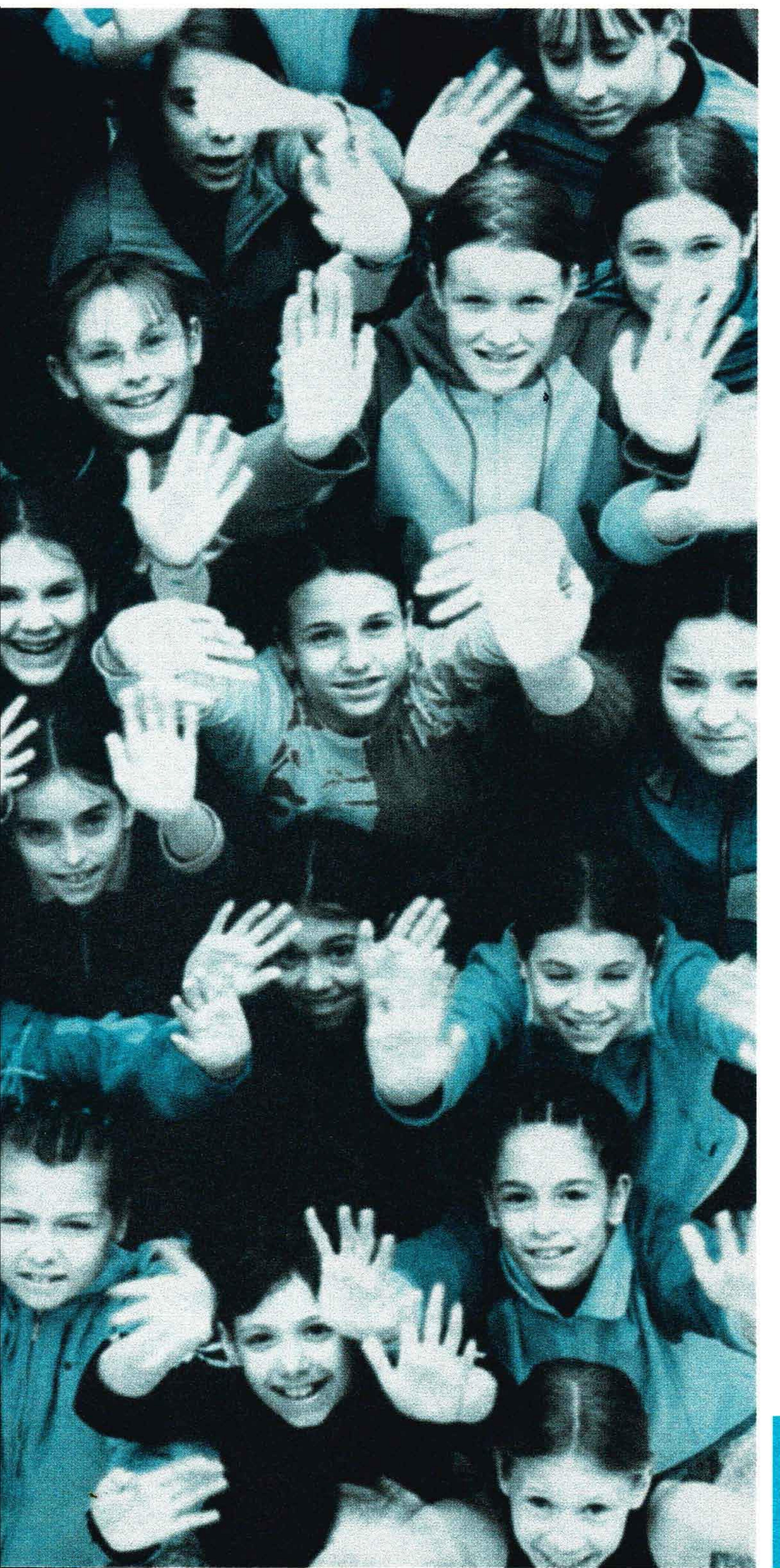

dos, se establecieron tres criterios básicos: la relación entre el indicador y las obligaciones inmediatas del Estado; que los indicadores aporten información sobre políticas públicas educativas en operación, o sobre la ausencia de políticas que sea necesario diseñar e implementar, con el fin de avanzar en la plena vigencia del derecho a la educación; y, finalmente, la posibilidad de obtener, en el corto y mediano plazo, información para su cálculo? .

En cuanto al primer criterio, se trata de identificar las principales obligaciones de carácter inmediato del Estado frente al derecho a la educación. Una vez identificadas, se eligen las que, de acuerdo con las Observaciones Generales 3a, 11 y 13 del Comité de Derechos Económicos, Sociales y Culturales, y las observaciones de la relatora especial de las Naciones Unidas para el Derecho a la Educación en sus informes 1998-2004, puedan ser entendidas como obligaciones que sin cumplimiento no se entienden, dadas las condiciones para el pleno disfrute al derecho a la educación. Tales obligaciones podrían ser comprendidas, en el ámbito de la política educativa, como las obligaciones básicas; es decir, el mínimo que las políticas educativas deberían garantizar. Lo anterior, sin desmedro de que para las obligaciones consideradas no básicas -como las de cumplimiento progresivo- el Estado adelante medidas de política pública para su cumplimiento.

9 Algunos de los indicadores propuestos se elaboran tomando como referencia la investigación titulada Sistema de seguimiento y evaluación de la politica educativa a la luz del derecho a la educación. Defensoría del Pueblo. Investigación realizada por Luis Eduardo Pérez Murcia. Bogotá, Colombia. 2004. pp. 40-44 y 78-128. 
Con relación al segundo criterio, los indicadores serán elegidos en función de las principales metas que, conforme al contenido del derecho a la educación, las políticas públicas deben garantizar. Esto, tomando como modelo de referencia el sistema de las 4-A, propuesto por la profesora Tomasevski, presentado en el acápite tercero.

Por último, en cuanto tiene que ver con la disponibilidad de información, se seleccionarán indicadores para los que existan fuentes de información de validez y confiabilidad reconocida, como las estadísticas producidas por las oficinas nacionales de estadística -para el caso de Colombia, el DANE-; las estadísticas de centros de investigación en educación, nacionales e internacionales; las estadísticas de centros especializados en información estadística, como el Instituto de Estadísticas de la UNESCO; las estadísticas producidas por organismos multilaterales, como el Banco Mundial, Banco Interamericano de Desarrollo y Comisión Económica para América Latina y el Caribe, CEPAL; las estadísticas oficiales presentadas por el Ministerio de Educación y las entidades territoriales competentes -las Secretarías de Educación para el caso de Colombia-, $y$, finalmente, la información reportada por los organismos centrales y regionales de planeación -Departamento Nacional de Planeación y secretarías de planeación, en Colombia-.

Es oportuno mencionar que existen obligaciones básicas para las que estas fuentes no reportan información con el nivel de desagregación necesaria para evidenciar posibles fenómenos de discriminación. En tal caso, es necesario que las entidades competentes incorporen los criterios según motivos de discriminación prohibidos para las variables pertinentes, así como se establece en el siguiente modelo:

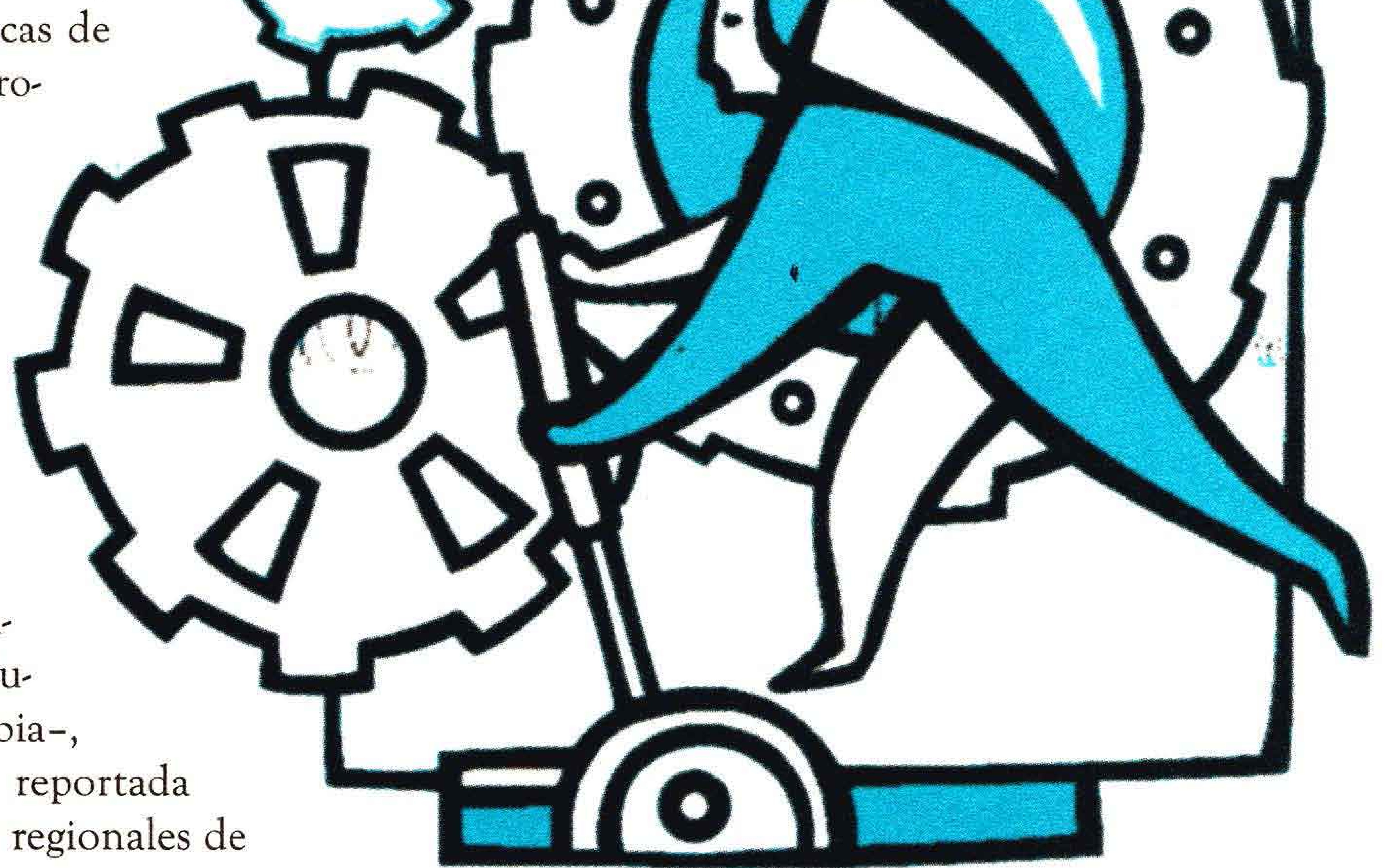


Asegurar el número de cupos equivalentes al número de niños y niñas en edad de enseñanza primaria.

Asegurar adecuadas
condiciones de
infraestructura física y
ambiental de las
instituciones educativas y
los centros de
enseñanza.

Asegurar la disponibilidad de docentes y la existencia de programas de instrucción académica y formación para los mismos.
Niños y niñas en edad escolar que no disponen de cupos escolares en el sistema público y que no disponen de recursos para ingresar en el sistema privado

Las inadecuadas condiciones te infraestructura de algunos establecimientos educativos ponen en riesgo la salud y la integridad física de los niños y las niñas.
- Déficit de cupos escolares. Localidad ${ }^{10}$.

- Nuevos cupos escolares Zona. creados en el sector oficial. Nivel educativo.

- Cupos contratados con el sector privado.

Motivos de discriminación prohibidos ${ }^{11}$.

Localidad.

Zona. vos que no disponen de impide que los niños y niñas dispongan, en forma permanente, de profesionales a cargo de su proceso de aprendizaje. Como consecuencia de la falta de docentes, en ocasiones se amplía la relación alumnodocente, a niveles que pueden afectar la calidad de la enseñanza. servicios públicos domiciliarios completos.

- Establecimientos educativos con infraestructura que amenaza ruina -paredes y techos en riesgo de desplomarse-.

- Establecimientos educativos ubicados en zonas del alto riesgo ambiental.

- Déficit de docentes en establecimientos oficiales.

Tipo de servicio del que no se dispone.

Localidad.

Zona.

Localidad.

Zona.

Tipo de riesgo.

Localidad.

Nivel educativo.

- Total de docentes que recibieron cursos de formación y actualización en un periodo de referencia.

- Relación alumno- docente.
Localidad.

Nivel educativo.

Sector.

Nivel educativo.
Sistema de información del Ministerio de Educación Nacional, MEN, y de las

Secretarías de Educación (SE).

DANE C-100.

Sistemas de información de MEN y SE.

Sistemas de información de MEN y SE.

DANE C-600.

Sistemas de información de MEN

y SE.

Sistemas de información de MEN y SE.

DANE.

\footnotetext{
10 Si las estadísticas son tomadas a nivel nacional, en lugar de localidad es pertinente desagregarlas para departamentos y municipios.

11 Discapacidad auditiva, discapacidad visual, retraso mental,síndrome de down, trastornos .
} 


$\begin{array}{ccc}\text { Obligación } & \text { Problema de Política Pública } & \text { Niveles de } \\ \text { Desagregación Fuente de Información }\end{array}$

Velar por el derecho de acceso a las instituciones y programas de enseñanza públicos, sin discriminación alguna.
Los grupos poblacionales discriminados, en especial, población con discapacidad, negra, indigena y desplazada, presentan niveles de matrícula y asistencia escolar por debajo del promedio del resto de la población.

Proporcionar educación básica, pública, obligatoria y gratuita para todos los niños y las niñas, y asegurar el acceso a la educación primaria, a toda la población.

Asegurar accesibilidad
económica a la
enseñanza básica,
mediante la aplicación
inmediata de su
gratuidad y asegurar la
accesibilidad física y
geográfica.

La educación primaria no es universal, y no se cumple con el principio de gratuidad.

Los grupos poblacionales de menores recursos, 0 en situación de pobreza, enfrentan la dualidad entre llevar a los niños y niñas a estudiar 0 satisfacer otras necesidades.

Los grupos poblacionales discriminados enfrentan barreras económicas, geográficas, sociales y culturales para acceder al sistema educativo.
- Tasa de cobertura bruta.

- Tasa de cobertura neta.

- Tasa de asistencia bruta.

- Tasa de asistencia neta.

Sector.

Nivel educativo.

Localidad.

Motivos de

discriminación

prohibidos.
C-600, Encuesta Nacional de Hogares (ENH) y sistema de información del MEN y las SE.
Sistema de información de las SE. matriculados en primaria, que reciben educación gratuita.

Porcentaje de la población mayor de 11 años que tiene nivel educativo la primaria completa o un nivel educativo mayor.
Sector.

Localidad.

Estrato socioeconómico. Motivos de discriminación prohibidos.

Motivos de discriminación prohibidos.
ENH y Encuesta de Calidad de Vida (ECV).
Sector. Valor promedio de la canasta educativa financiada por los padres.

- Porcentaje de los gastos familiares destinados a la educación de los niños y las niñas.

- Inversión anual del Estado por habitante en educación.

- Porcentaje de la población estudiantil que estudia en una localidad distinta a la de residencia.

- Distancia a la escuela expresada en minutos.
Estrato socioeconómico.

Nivel educativo.

Sector.

Localidad.

Nivel educativo.
ECV.

DNP - Misión Social.

Sistema de información del MEN.

Sistema de información de las SE.

Sistema de información de las SE. 
Obligación

Derecho de Acceso a la Educación. Obligación de Accesibilidad

Implantar un sistema adecuado de becas para las poblaciones más vulnerables.

Erradicar el analfabetismo.
Los sistemas de subsidios y becas son insuficientes frente a la magnitud de población que no tiene cómo pagar la educación de los niños $y$ las niñas y tiene que retirarlos delasistema educativo.
- Porcentaje de estudiantes que disponen de becas de estudios.

- Inversión del Estado en subsidios educativos.

- Inversión promedio en educación por contratación de cupos educativos en el sistema no oficial.

- Total niños y niñas beneficiarios de los programas de becas contratadas con el sistema educativo no oficial.

\section{Estrato}

socioeconómico.

Sector.

Motivos de discriminación prohibidos.

Nivel educativo.
DANE-ECV.

Sistema de información del MEN y las SE.

Sistema de información del MEN y las SE.

Sistema de información del MEN y las SE.
Zona

Motivos de

discriminación

prohibidos.
DANE - ENH y ECV. alfabetización universal y

existen diferencias

notables entre las tasas

de analfabetismo urbano

$y$ rural.
- Tasa de analfabetismo. ridad. n 
Establecer y garantizar que se cumplan normas mínimas de enseñanza en las escuelas públicas y privadas, y ejercer la inspección y vigilancia del sistema educativo en todo el territorio y en todos los niveles educativos, con el fin de velar por su calidad.
Los resultados de pruebas nacionales e internacionales de calidad de la educación evidencian que los niños y las niñas no alcanzan niveles mínimos de competencia en áreas básicas del conocimiento, - Tasa de estudiantes gracomo lenguaje, matemáticas y sociales. dad para cursar primaria. duados, con relación al
Vigilar y garantizar que la Persisten formas de disciplina escolar sea compatible con la dignidad humana. castigo corporal y psicológico, como mecanismo para promover el orden y la disciplina escolar.
- Tasa de promoción escolar. Sector.

- Años promedio de escolari- Nivel educativo.

- Años promedio de escolaridad para cursar secundaria. total de estudiantes matriculados en un período académico.

- Resultados de pruebas de competencias básicas.

- Resultados de pruebas del ICFES.

- Tamaño promedio de los grupos.

- Quejas interpuestas por estudiantes y/o padres de familia ante MEN o SE, relacionadas con maltrato físico o psicológico en los establecimientos educativos, durante un período de referencia.
Localidad.

\section{Sector.}

Estrato socioeconómico.

Sector.

C-600.

Sistema información MEN y SE.

Sistema información MEN y SE.

Estrato socioeconómico.

Sector.

Estrato

Sistema información MEN y SE.

ICFES.

socioeconómico.

Sector.

Nivel educativo.

Estrato socioeconómico.

Sector.

Nivel educativo.

Estrato

socioeconómico.

Sector.

Nivel educativo

Sector.

Nivel educativo.

Estrato

Sistema información

socioeconómico.
ICFES.

Sistema información MEN y SE. 
Velar por que el principio de libertad no genere desigualdades en la calidad de la educación que reciben los grupos poblacionales más vulnerables.

Mejorar de manera continua la capacidad intelectual del cuerpo docente.

\section{Existen notables} diferencias en el nivel de competencia que se adquiere en el sistema educativo público y privado de alto nivel; en especial, los colegios internacionales.

Docentes sin título profesional y, lo que es más preocupante, sin bachillerato completo, imparten enseñanza. Lo anterior, sin que exista una estrategia de alcance nacional y local, para mejorar el nivel educativo de los docentes.
- Resultados de pruebas del Icfes.

- Estudiantes admitidos a las universidades públicas.

- Porcentaje de docentes que tienen título profesional.

- Porcentaje de docentes que tienen como máximo nivel educativo la secundaria completa.

- Porcentaje de docentes que tienen como máximo nivel educativo la primaria completa.

- Total docentes que recibieron cursos de formación y actualización en un período de referencia.

\section{Estrato} socioeconómico.

Sector.

Estrato socioeconómico.
Icfes.

ICFES y Sistema de Información del MEN.

Sector Zona.

Sector.

Zona.

Sector.

Zona.

Sector.

Zona.

Sector

Zona.
Sistema de información del MEN y SE.

Sistema de información del MEN y SE.

Sistema de información del MEN y SE.

Sistema de información del MEN y SE.

Sistema de información del MEN y SE.

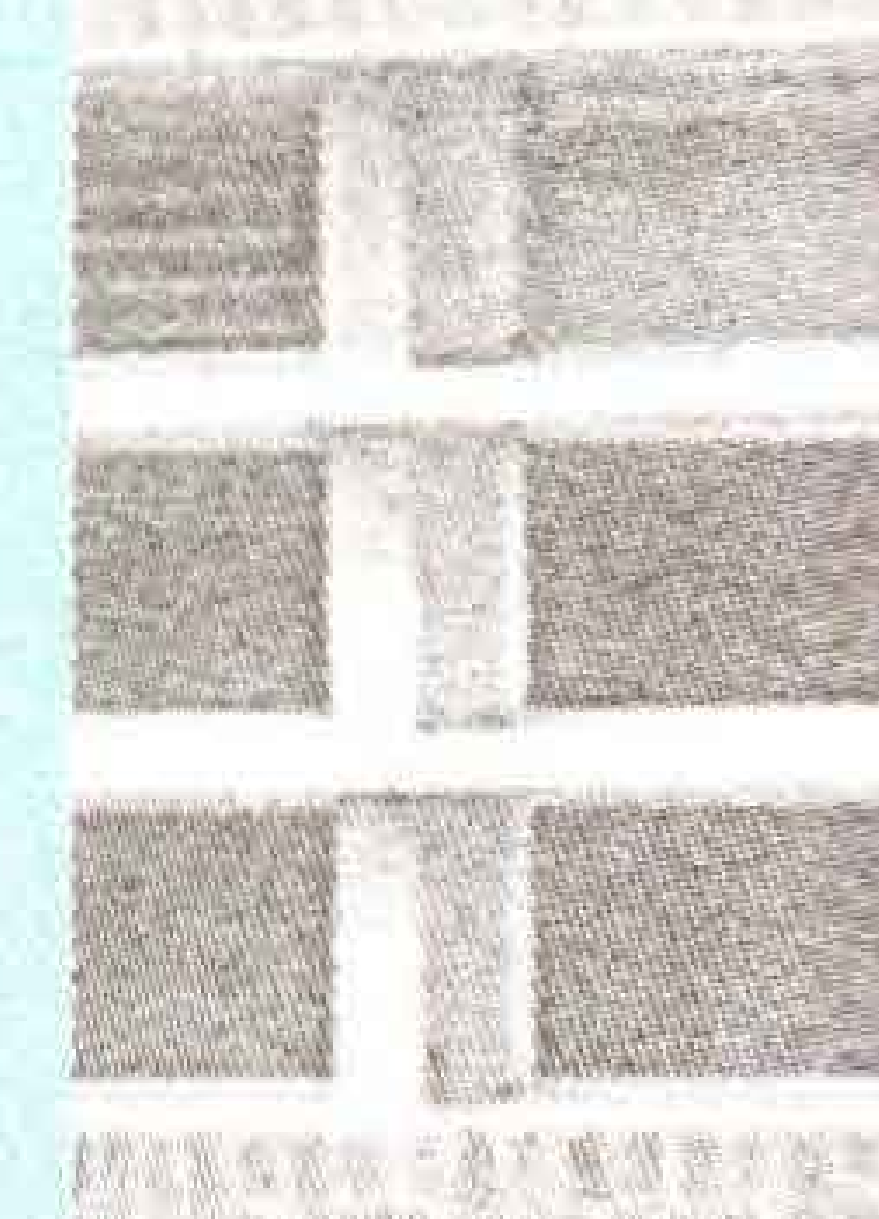

5xis

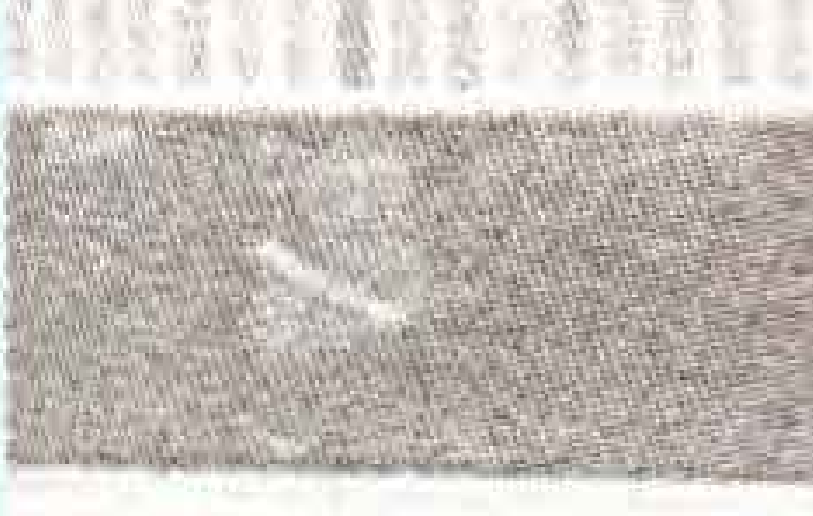

- Resultados de pruebas de evaluación docente. 


$\begin{array}{ccc}\text { Obligación } & \text { Problema de Politica Pública } & \text { Niveles de } \\ \text { Desagregación Fuente de Información }\end{array}$

Asegurar la permanencia Existen altas tasas de de los niños y niñas en la deserción escolar y es educación pública, básica, obligatoria y gratuita, y adoptar medidas para fomentar la asistencia regular a las escuelas.
Velar por la prestación eficiente y continua del servicio público educativo (CPC, Art. 365; CC, Sentencia T-5161996, Sentencia T-5711999).

Prohibir y eliminar toda forma de discriminación que atente contra la permanencia de los niños y las niñas en el sistema escolar. Asegurar que la disciplina escolar sea compatible con la dignidad humana, la igualdad de trato y el libre desarrollo de la

personalidad, y asegurar el debido proceso en la imposición de sanciones. evidente la ausencia de medidas orientadas a fomentar la permanencia de los niños y las niñas en la escuela.
- Tasa de deserción escolar.

- Tasa de asistencia escolar. escolar a quinto grado.

- Tasa de supervivencia escolar a once grado.

- Esperanza de vida escolar.

- Subsidios educativos para promover la permanencia escolar.

Existen múltiples motivos por los que no se asegura la prestación

continua del servicio público educativo; entre ellos, cabe mencionar el paro de docentes, la falta de servicios públicos y las expresiones del conflicto armado interno.

\section{Coexisten múltiples} formas de discriminación en la escuela, que provocan la deserción escolar de ciertos sujetos y grupos poblacionales, en especial niñas gestantes, personas con discapacidad, personas con tradición lingüística propia, entre otros grupos.
Días de suspensión de clases al año.

- Existe un proyecto o programa orientado a asegurar la permanencia de los niños y las niñas en el sistema educativo.

\section{Número de niños y niñas} educativo y que se reintegraron en un período de referencia, como resultado del proyecto o programa orientado a asegurar la permanencia de los niños y las niñas en el sistema educativo. que desertaron del sistema

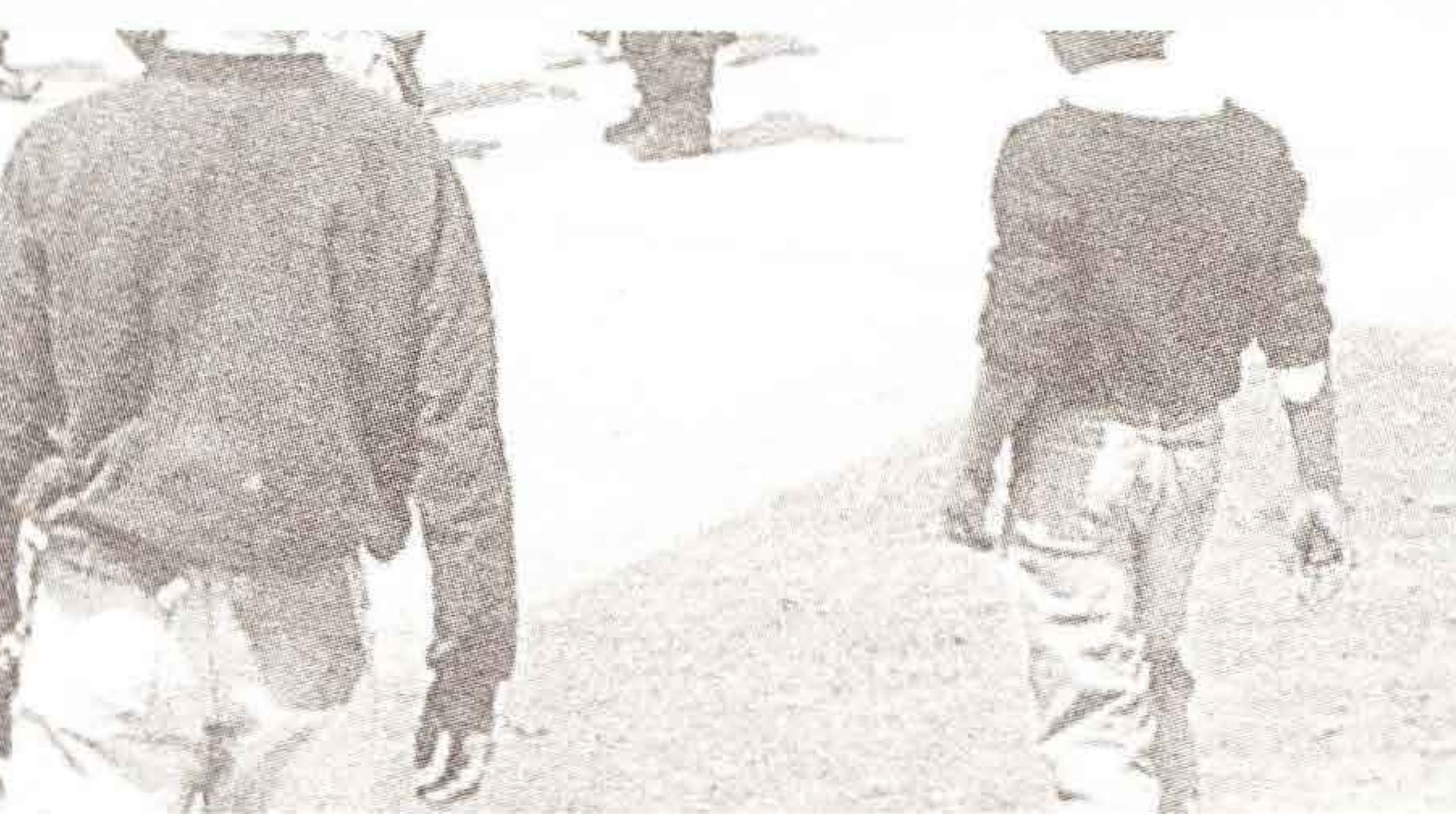

Sector.

Nivel educativo

Motivos de

discriminación

prohibidos.

Sector.

Zona.

Nivel educativo.

Motivos de

discriminación

prohibidos.

Sector.

Causas de suspensión.
C-600.

Sistemas de información del MEN y SE.

UNESCO.

Sistema de información del MEN y SE.

Sistema de información del MEN y SE.

Sistema de información del MEN y SE.

Localidad.

Nivel educativo.

Motivos»de

discriminación

prohibidos.
Sistema de información del MEN y SE.
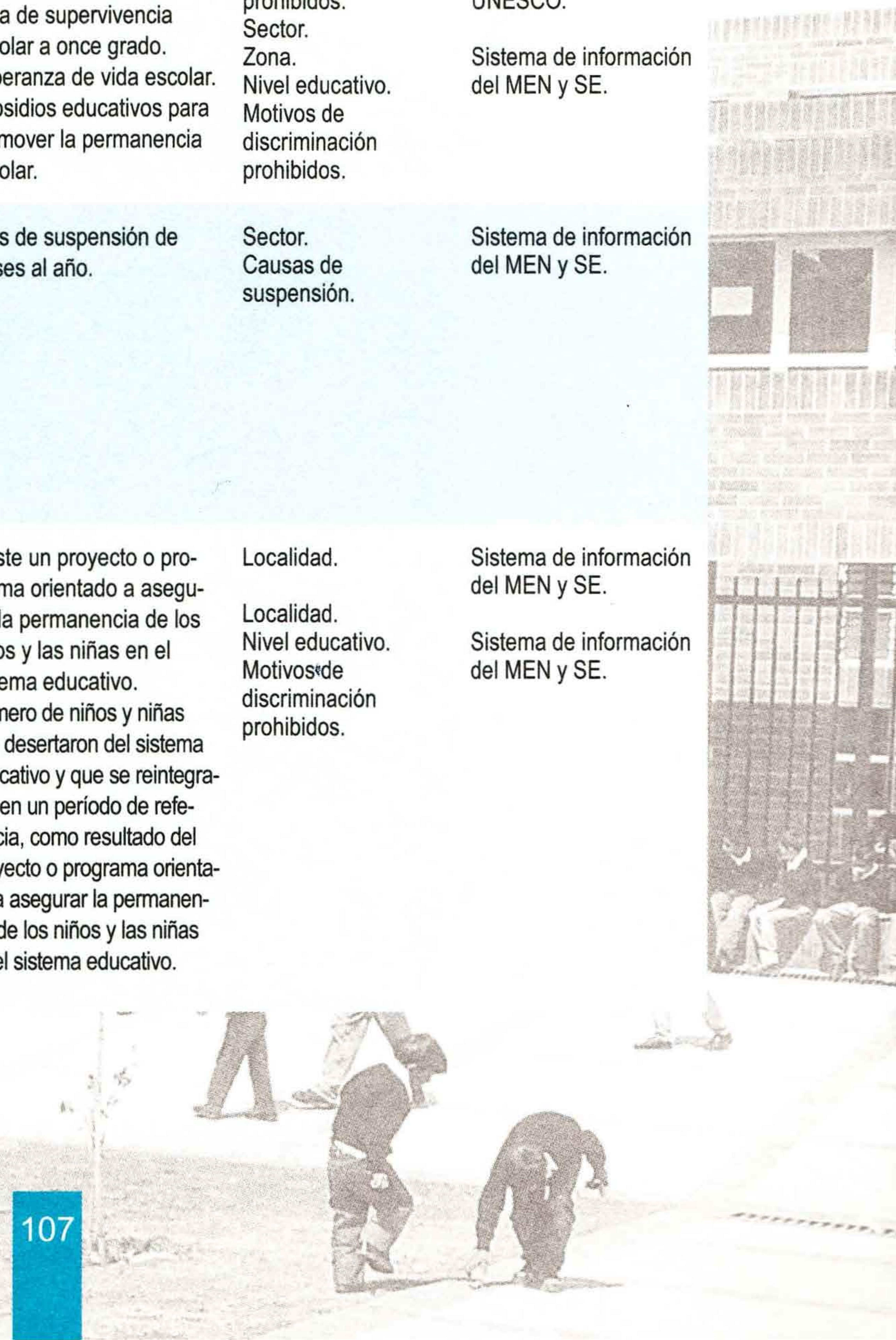


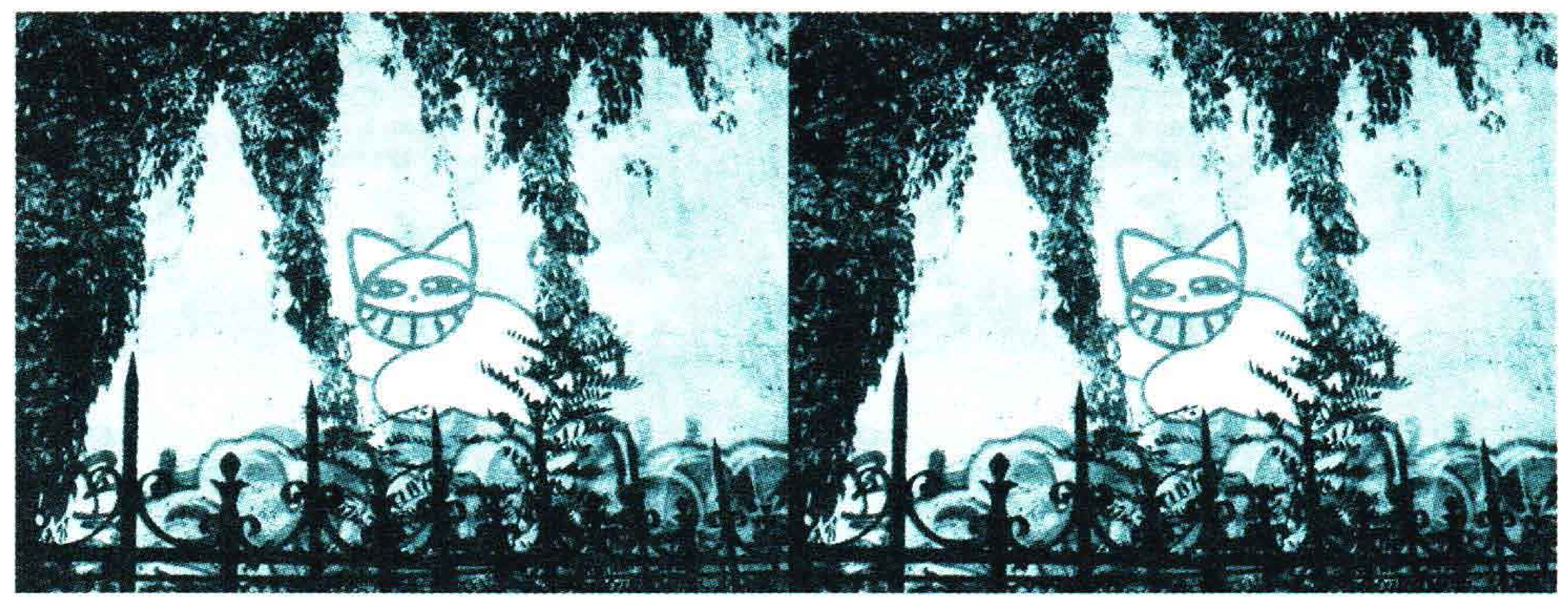

\section{Obligación \\ Problema de Politica Pública \\ Indicador \\ Niveles de \\ Desagregación \\ Derecho a Permanecer en el Sistenta Educativo, Obligación de Adaptebilidad}

Fuente de Información

Asegurar que en los centros educativos públicos y privados se imparta la enseñanza que mejor se adapte a los niños y las niñas.
Las escuelas no se adaptan, ni en lo pedagógico ni en lo físico, a las necesidades especiales de aprendizaje de los grupos poblacionales vulnerables; en especial, personas con discapacidad, personas con tradición lingüística propia; personas privadas de la libertad.
- Establecimientos educativos especializados en la atención de niños y niñas con discapacidad.

- Establecimientos educativos que integran niños y niñas con discapacidad.

- Establecimientos educativos que integran niños y niñas con discapacidad, que disponen de docentes especializados.

- Establecimientos educativos que integran niños y niñas con discapacidad, que disponen de apoyos especializados.

- Establecimientos educativos que ofrecen programas etnoeducativos.
Sector.

Nivel educativo.

Tipo de discapacidad.

Sector.

Nivel educativo

Tipo de discapacidad.

Sector.

Nivel educativo.

Tipo de discapacidad.

\section{Sector.}

Nivel educativo.

Tipo de discapacidad.

Sector.

Nivel educativo.
Sistema de información del MEN y SE. 


\section{Tabla 2: modelo de indicadores desagregados, según obligaciones y problemáticas del sector educativo}

Es importante mencionar que, una vez se disponga de la información para los diferentes indicadores que integran los componentes del derecho, es posible elaborar un indicador compuesto para cada uno de éstos $y$, posteriormente, un indicador que combine los diferentes componentes, con el fin de tener un único valor que dé cuenta del grado de avance en el nivel de satisfacción del derecho a la educación preescolar, básica y media.
Dicho indicador puede ser llevado a una escala entre cero y cien puntos, de tal manera que, a mayor puntaje, mayor nivel de realización del derecho a la educación. El indicador tendrá la virtud de evidenciar en cuál de los componentes del derecho a la educación se ha avanzado menos, calcular el déficit para alcanzar las metas por componente -distancia entre el puntaje obtenido y cien-y, además, arrojar información sobre la magnitud del déficit a nivel global, para alcanzar la meta de realización del derecho a la educación de todos y todas, sin discriminación.

\section{Bibliografía

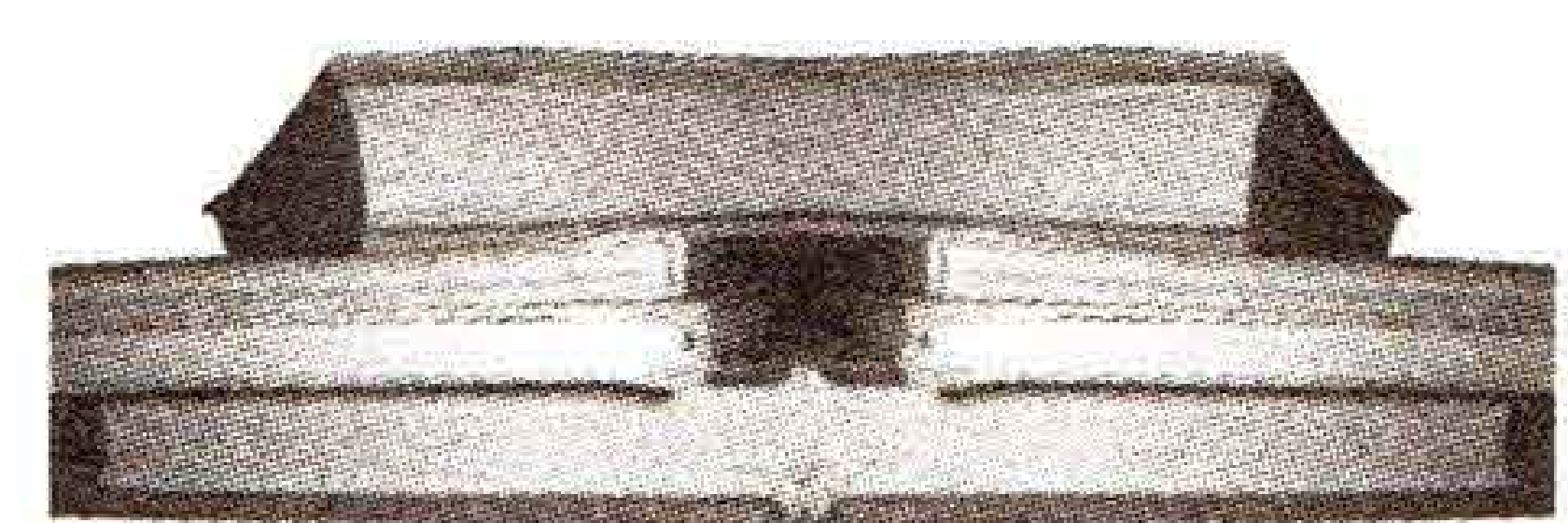

Amartya K. Sen. "Los bienes y la gente", en revista Comercio exterior, vol. 33, número 12, México, diciembre de 1983.

Comité de Derechos Económicos, Sociales y Culturales. Observación General No. 3, La indole de las obligaciones de los Estados Parte, Documento E/1991/23, Quinto período de sesiones, 1990.
Comité de Derechos Económicos, Sociales y Culturales. Observación General No. 11, Planes de acción para la enseñanza primaria, Documento E/C.12/1999/4, 20 período de sesiones, 1999.

Comité de Derechos Económicos, Sociales y Culturales. Observación General No. 13, El derecho a la educación, Documento E/ C.12/1999/10, $21^{\circ}$ período de sesiones, 1999. 
Departamento Nacional de Planeación. La educación en cifras, boletín No. 19, Bogotá, Colombia, 1999.

Organización de las Naciones Unidas para la Educación, la Ciencia y la Cultura. Informe de seguimiento a la EPT en el "mundo 2005, Educación para todos: el imperativo de la calidad, UNESCO, París, Francia, 2004.

Pérez Murcia, Luis Eduardo. Sistema de seguimiento y evaluación de la política educativa, a la luz del derecho a la educación, Defensoría del Pueblo, Bogotá, Colombia, 2004.

Gonzáles Julia Elizabeth, Sabogal Carlos. La integración educativa de los niños y las niñas con discapacidad: una evaluación en Bogotá, desde la perspectiva del derecho a la educación, Defensoría del Pueblo, Bogotá, Colombia, 2004.

Programa de las Naciones Unidas para el Desarrollo. Informe sobre Desarrollo Humano 2000, Derechos humanos y desarrollo humano, Nueva York, 2001.

Programa de las Naciones Unidas para el Desarrollo. Informe sobre Desarrollo Humano 2005. La cooperación internacional ante una encrucijada: ayuda al desarrollo, comercio y seguridad en un mundo desigual, Ediciones Mundi-Prensa, Nueva York, 2005.

Sarmiento Gómez, Alfredo. "El uso de encuestas e indicadores sociales", en Indicadores sociales y de gestión, DANE, Bogotá, 1995.

Perla Tovar, Luz, Alam,

Carmen. Situación de la educación básica, media y superior en Colombia, Corpoeducación, Educación, Compromiso de Todos, Casa Editorial el Tiempo, Fundación Corona, Fundación Antonio Restrepo Barco, Bogotá, Colombia, 2002.

Tenjo Galarza, Jaime. "Hacia el desarrollo de indicadores en el área educativa", en Indicadores sociales y de gestión, Departamento Administrativo Nacional de Estadísticas, Bogotá, Colombia, 1995.

Tomasevski Katarina. Human Rights Obligations: Making Education Available, Accessible, Acceptable and Adaptable, Education Primers No. 3, Novum Grafiska AB, Gothernburg, Suecia, 2001. 